\title{
Traditional Catering Industry Carries Cooperative Robots to Achieve Industrial Upgrading
}

\author{
Zhang zhongyu* \\ School of Public Administration and Service, Lecturer of Shanghai Urban Construction Vocational College \\ Shanghai, China
}

\begin{abstract}
The world-renowned Chinese cuisine has been handed down from generation to generation in the traditional mode of handicraft, workshop and apprenticeship, which is distorted from generation to generation. The quality of dishes in small shops is difficult to guarantee, the works of masters are difficult to replicate, economies of scale are difficult to achieve, waste of resources can not be controlled, and the plight of industrialization has not been broken through. In recent years, with the development of science and technology, especially the growing maturity of cooperative robots, the traditional catering industry has ushered in an opportunity for innovation. Through the application research of multi-joint cooperative robot in Chinese cooking scene, this paper solves the technical difficulties of large-scale mechanization of Chinese cooking, opens up the last kilometer of Chinese food industrialization, realizes the upgrading of traditional catering industry, and also enables the inheritance and innovation of Chinese cooking.
\end{abstract}

\section{Development Status of Chinese and Western Food and Beverage Industry}

\subsection{Current Situation of China's Catering Industry}

China's catering industry has a long history, and the market scale is growing steadily, from 2892.6 billion yuan in 2014 to 4672.1 billion yuan in 2019, with a compound annual growth rate of $10.1 \%$, and is expected to reach 7 trillion yuan in 2025. The market demand is sufficient, and the huge catering market in China not only provides a huge potential for the development of the industry, but also gives a new opportunity for the times.

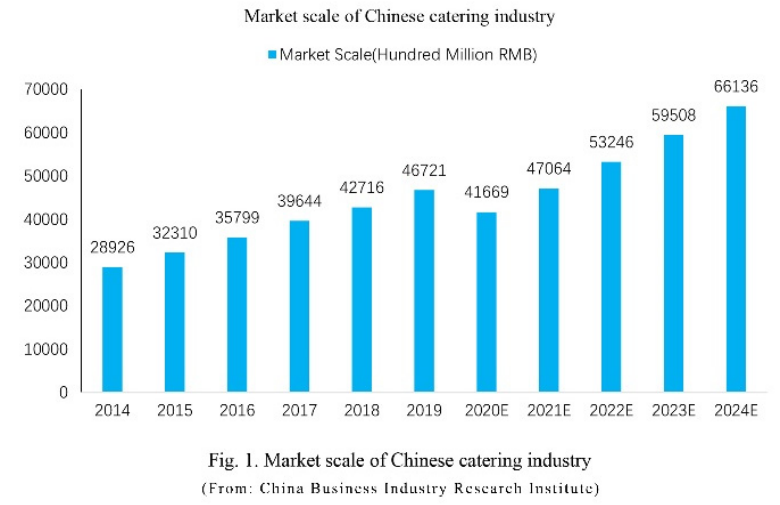

Statistics to 2019 , the market size of Chinese food accounted for $80 \%$, and Western food accounted for $20 \%$. In China's Western food market, it has a strong inclusiveness. In addition to fast food, coffee and bars, it also absorbs French food, Italian food, Russian food, American food, German food, Japanese food, Korean food and Turkish food. There are more than 30,000 restaurants, and the number of chain restaurants is increasing.

Market scale(By food type) of China's catering industry in 2019

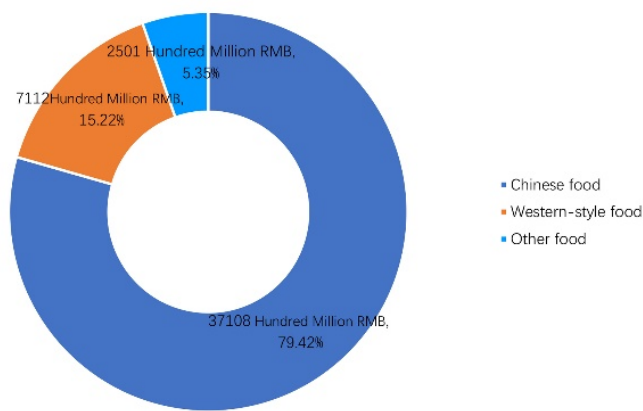
Fig. 2. Market scale(By food type) of China's catering industry in 2019

Chinese restaurants are divided into self-operated, franchise chain restaurants and non-chain restaurants according to their business model. Non-chain independent restaurants account for $80.4 \%$, reaching 3754.9 billion yuan in 2019. They have a large volume, but they have not been a big and strong leading enterprise. Chinese food industry belongs to the typical "big industry, small company". The largest Haidilao has a market share of only $0.3 \%$, and the total market share of the top five companies in the industry is less than $1 \%$. But in the United States, McDonald's alone accounts for more than $3 \%$ of the industry's sales.

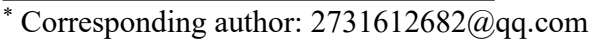


Market scale(By business model)of China's catering industry in 2019

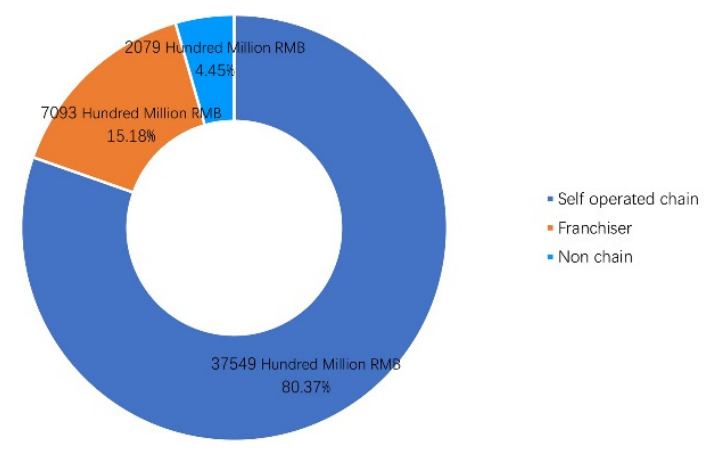

Fig. 3. Market scale(By business model)or China's catering industry in 2019 (From: China Business Industry Research Institute)

\subsection{Bottleneck of Chinese Food Development}

China has a vast territory, diverse climate, rich products and five thousand years of splendid civilization, which has bred a broad and profound food culture, and Chinese food culture is an important part of its civilization history. For thousands of years, nearly 100 kinds of cooking techniques, such as stir-frying, quick-frying, deep-frying, braising, quick-frying, simmer, sauteing, steaming and marinate, have formed different cuisines and varieties with different flavors. Chinese cooking, incomparably mysterious, with handicraft and workshop as the main mode, one person, one stove, one teacher and one art, even if the same cooking method still presents different tastes, it is difficult to achieve consistency in technology, mellow juice, fragrant glutinous meat is not greasy Dongpo Meat, the original flavor of tender and nourishing FeilongSoup, only the royal court food enjoyed by the royal family, hundreds of thousands of years. Through written records can not restore the true, difficult to copy, from generation to generation and distortion, there are a thousand hands, there are a thousand tastes.

The teaching of Chinese cooking has been continuing the inheritance mode of apprenticeship, following the traditional way of word of mouth and understanding. There is no uniform standard for the process of Chinese food production. Under the guidance of "a little salt, a proper amount of sugar, two spoons of vinegar and stirfrying with medium fire", Chinese food is made by experience, each shop doing things, which makes the inheritance of Chinese food skills fragmented and still stays in the development mode of folk crafts and folk workshops. Western fast food has entered China, and in just a few decades, KFC, McDonald's and Pizza Hut have spread all over major cities in China. The industrialized production of catering products has more than a hundred years of experience in foreign countries. Through largescale mechanized production, they can reduce production costs and improve labor efficiency. The main advantages are that the product quality is unified and stable, and the allocation of resources is optimized, so that Western food can be quickly replicated and promoted in China, showing the strong competitiveness of Western food industry, which is worth learning and learning from. This is precisely what Chinese food enterprises have not broken through and failed to do. Chinese food is permeated with the unique charm of Chinese culture from the selection of materials, cutting and matching, cooking, plating and dish names, and the color, fragrance and taste endow the dishes with personality and artistic conception. Every dish of Chinese food is like a work of art, which is also the obstacle to the scale of Chinese food. "Quantification of product standards" and "large-scale mechanized production" are the winning weapons to realize the industrialization of Western food. We can no longer "gnaw on the old" and rely on the cultural heritage left by our ancestors to eat, we should not only inherit but also innovate. The huge market prospect and strong sense of crisis force us to break the original inherent way and concept, abandon the original mode of front shop and back shop, and develop Chinese catering to industrial production, so the upgrading of traditional industries is imperative.

Faced with the differences in the development of Chinese and Western food brought about by the industrialization of catering, market forces have awakened, and in recent years, various market players have begun to explore and try the industrialization of Chinese food.

\section{Industrialization Process of Chinese Food}

\subsection{Misunderstanding of Catering Industrialization}

When it comes to the industrialization of catering, many people think that it is single and has no characteristics, but in fact it is not.

In the past hundred years, with the increasingly advanced technology and management, Western-style catering has become more quantitative, standardized, mechanized and intelligent in food processing. A variety of technologies such as bioengineering, flavor chemistry, food additives, nutrition and detection, technology and equipment, packaging and packaging materials, supply chain and information technology are comprehensively used to complete the industrialization of catering. It puts efficiency and safety in the supply chain, taste and flavor in the restaurant, for example, bacteria can't thrive in the frozen state, and rapid freezing makes food retain nutrients better. Industrial production, starting from raw materials, should be made from such processing workshops according to unified standards and processes, and then distributed to various stores, which not only ensures the safety and hygiene of food, but also ensures the unified taste and quality, so that consumers can eat at ease and rest assured.

After the mature western fast food entered China on a large scale, it broke the traditional mode of Chinese catering formed for thousands of years, and also set a benchmark for the industrialization of Chinese catering. Yum is one of the Fortune 500 catering companies in the world and the second largest franchise fast food group in the world, with 40000 restaurants in more than 100 
countries and regions around the world, of which about $2 / 3$ are located outside the United States. It is unimaginable to ensure that there is no standardization, so that no matter where you are, you can enjoy the taste in your heart. It is a great material and spiritual satisfaction.

However, the profound Chinese food takes thousands of years of catering culture as its length, full of distinctive cooking technology as its width, and broad and rich industrial chain as its depth. The three-dimensional industry of Chinese food has always provided infinite temptation and space for the market, and industrialization has made diversity and characteristics more guaranteed.

\subsection{What is Catering Industrialization?}

Industrialization is the replacement of manual labor by machine production, which represents an advanced mode of production and shows great power. The industrialization of catering industry, simply speaking, is to use modern science and technology, through largescale machine production, to achieve precise control of operation, ensure quality, reduce consumption and improve efficiency. The industrialization of Chinese food provides new momentum for the development of traditional catering, is the upgrading of the industry, and is also the direction of innovation and development of Chinese food, which is irreversible. The breadth and depth involved in the industrialization of Chinese food will trigger another industrial revolution.

\subsection{Exploration and Practice of Chinese Food in the Process of Industrialization}

Chinese food has also made many useful attempts and efforts in the past 30 years, and has generally experienced these aspects:

The rise of kitchen appliances: rice cookers, microwave ovens, ovens, steamers, bread machines and so on are the first attempts and partial completion of the standardization of certain specific food production, which are widely sought after by Chinese families and have been selling well.

With the progress of science and technology, especially the rise of robots, stir-frying machines, smart pots, smart restaurants and so on have sprung up one after another, and businesses have improved their products and technologies. By designing the mechanical movement and path of pots and pans and imitating the stir-frying of chefs, these have taken a step towards the digitalization and mechanization of Chinese food. However, these are focused on a product, a flash in the pan, failed to be promoted and popularized, product design is still deficient, failed to meet the needs of diversification of Chinese cooking.

Catering chain enterprises are booming for a while, and central kitchens are also booming. This is the catering enterprises in order to improve efficiency, from focusing on products to focus on business, from the management of standardization. Chain restaurants have adopted the central kitchen model to centralize and unify the processing of food materials. Now we see a lot of chain restaurant brands that promise 30-minute meals. The dishes served by those busy chefs on the bright kitchen stove are not entirely their skills. Most of them are semifinished products completed in the central kitchen. The store end is just hot processing, reorganizing the taste and ingredients, and completing the final production. Xiaonanguo, which was very popular for a time, openly "admitted" that $95 \%$ of its own dishes had been industrialized, and that the delicious Black Truffle River Shrimp, Sweet and Sour Mandarin Fish and Braised Pork Louver Knot you ate were all processed and made in the central factory.

Chafing dish chain is the simulation and pioneer of Chinese food industrialization. The standardization of Chinese food is not easy, the pace is heavy and difficult, where to break through and practice? As a subdivision of Chinese food, hot pot has its unique advantages in standardization: first, it does not rely on chefs, the characteristics of hot pot are how to do, how to eat, customers have the final say, customers are chefs; second, the store provides standardized raw materials, which are relatively simple; third, the acceptance is high, and you can adjust the dipping sauce to taste what you want to eat. These points determine that in the Chinese food industry, hot pot is the easiest to achieve scale, and the data also support this point: hot pot has become the largest category of Chinese food with $13.7 \%$ of catering consumption. Hotpot industry can be used as a pioneer of large-scale standardization of Chinese food industry, and its experience and lessons can be used for reference.

Whether it is kitchen appliances, intelligent wok, the implementation of the central kitchen chain catering or the highest proportion of chafing dish meals, have made a useful attempt for the industrialization of catering, accumulated valuable experience, but Little Sheep, Xiaolongkan, Haidilao, Xiaonanguo, also did not complete the historical mission of the industrialization of catering, chafing dish bypassed the Chinese cuisine. Only in the preparation of meals, management and other aspects to achieve the standard and scale, so the reference and experience is far from enough, where is the difficulty?

\section{The key Analysis of Chinese Food Industrialization}

To realize industrial production, we must first realize "standardization" and "mechanization". Among them, standardization includes three aspects: standardization of raw materials, standardization of production process, standardization of quality and hygiene of finished products. The premise of all standardization is to quantify, that is, digitalization. Chinese food has been based on experience, apprenticeship small-scale teaching model, which is the fatal point of Chinese food production can not be large-scale, therefore, let Chinese food from raw materials to technology to the whole process of digitization, is the basis and prerequisite for the industrialization of Chinese food. 


\subsection{Digitization Standard of Raw Materials}

Chinese food is mainly agricultural products, different origins and different textures, the difficulty of standardization of Chinese food increases, so it is necessary to start from the standardization of raw material types to the production and breeding process, such as the establishment of a unified mariculture base, agricultural production base, vegetable cultivation base, etc. Unified varieties, unified production standards, unified management mode, unified collection standards, unified selection standards, etc; On this basis, the establishment of a unified and centralized industrial processing base, raw materials into semi-finished products, are the feasible way to quantify the standard of raw materials, this is not difficult, there are many such bases.

\subsection{Digital Standardization of Cooking Process}

There has been no uniform standard for Chinese cooking. On the issue of standard quantification, the old tradition has been continued. Descriptive words such as "a little salt, a proper amount of sugar, a spoon of vinegar, stir-fried over medium fire" guide the operation and make people feel at a loss. From standardization, specific numbers and physical units must be used to specify the quantity, weight, temperature, humidity and pressure. At present, in the multimedia environment, text, image, audio and video can be used comprehensively to record the operation process of chefs and the wrist action of cooking in a multi-angle, all-round and whole-process manner, and all information and processes such as food materials, seasonings, temperature, techniques, actions, procedures, time and pots can be recorded holographically and converted digitally.

Industrial production is to speak with figures and minimize human factors, which can be standardized. Completed the cooking process of digital quantitative standards, or can not guarantee the standardization of products, because chefs can not be uniform, everyone is different, techniques, emotions, movements, taste are different, industrialization must be standardized machine production, that is, people to change into machines. This is also the biggest obstacle and the last step in the industrialization of Chinese cooking.

Today, with the development of science and technology, this problem has finally made a breakthrough.

\subsection{Cooperative Robots are the Key to the Industrialization of Chinese Cooking}

In the past ten years, robotics technology and industry have developed rapidly in the world, the degree of intelligence is getting higher and higher, and the application field is expanding. Since 2016, with the support of big data, cloud computing and other technologies, the artificial intelligence industry has exploded, helping the industrial robot industry to open a new era, human-computer cooperation and humancomputer integration have become the mainstream trend of the market, constantly pushing cooperative robots to the market outlet [3]. China has been the world's largest industrial robot market for six consecutive years, and the application industry has expanded to 47 industries and 129 industries in the national economy. In this context, the State Council issued "Made in China 2025" and began China's "industry 4.0" development strategy. Intelligent manufacturing is the core of China's industrial transformation and upgrading, and "Internet + " and robotics technology are effective means and tools to achieve intellectualization. In the future, robots will do all the work that is not suitable for human work, bad conditions, dangerous, complicated, standardized and repetitive. The application of robots has rapidly expanded from manufacturing industry to various service industries, and the first floor sweeping robots, food delivery robots, escort robots, education robots, rehabilitation robots and supermarket robots in the market have entered daily life. The service areas and objects of robots have been expanding, and their applications have penetrated into many fields.

Cooperative robot is an intelligent robot which has the ability of automatic control and programming, and can interact and cooperate with human beings in the same workspace. Compared with traditional industrial robots, cooperative robots break the limitation that they can only be used in industrial scenarios. With the advantages of low weight, high flexibility, easy programming, rapid configuration and small limitation of operation space, cooperative robots have broad application prospects in medical, education and training, new retail and other service scenarios.

Multi-joint robot refers to the machine imitating human joints and fingers to complete fine movements. Dozens of motors, joints and hundreds of sensors are installed on the arm of the robot. Its "joints" are as flexible as human beings, and its strength and speed can accurately imitate the movements of human hands.

Throughout Chinese cooking seems complex, in fact, dozens of commonly used operations are decomposed one by one, not the need for precise to micron fine movements and difficult movements, all the actions of chefs, mostly using large joints, arm operation, which for multi-joint cooperati ve robots, completely without obstacles and difficulties, according to data. It is replaced by intelligent cooking robots or kitchen actuators. Because it is operated by machine, it has a high degree of standardization, which ensures the high quality of dishes and can be copied tirelessly.

\subsection{Application Analysis of Multi-joint Cooperative Robot}

The multi-joint cooperative robot has high technology content and high development cost, and is further promoted in the fields with high added value such as medical treatment at present. A surgeon needs to accumulate many years of clinical experience, and the technical requirements and the labor cost of the surgeon are very high. The multi-joint cooperative robot can replace a surgeon to accurately and stably complete an operation, and has high cost performance. However, if it 
is used to replace manual labor with low hourly wage such as assembly, packaging, handling and express delivery, the input-output ratio is low, so the market promotion and application are limited. In the field of catering, in the cooking process, the multi-joint robot can accurately pick up materials, cut oil, cut vegetables, add materials, stir fry, turn the pot, take out of the pot, wash the pot and grab all kinds of bottles, tableware, pots, use mixers, coffee machines, ovens, steamers and other supporting kitchen equipment and instruments, which is almost all the actions and work needed for cooking. Cooperative robots also have more and more intelligent functions such as autonomous learning, cooperation with people, adaptation to complex environments and so on. Over the past three years, with the development and application of multi-joint robots and collaborative robots, it has been fully competent for all the actions of master-level intelligent kitchen and replicated chef works, saving labor costs for business owners and winning public praise. The key is that the catering industry is large in scale and there are tens of millions of chefs, so the application prospects are considerable.

Multi-joint robot in the inspection of finished products, standard quantification is equally important, in addition to intuitive sensory evaluation, must also increase physical and chemical indicators, microbial indicators, these rely on advanced testing equipment and strict testing means to achieve, in order to ensure the authenticity, accuracy and reliability of data, as a basis for product quality control.

Standardized food materials, standardized procedures, standardized condiments and standardized robotic operation can lead to safe, hygienic and stable finished products in a real sense. The whole process of cooking is quantified by numbers and the standard is unified, so as to ensure that the quality of dishes will not be affected by the differences of raw materials, being in different places, the level of chefs, today's mood and other factors, and ultimately ensure that the dishes are of the same high quality. Future robots will become more and more intelligent, carrying cloud computing, 5G communications, big data and industrial Internet and other important technologies, to provide robots with a more intelligent cloud brain, the "cloud chef", in fact, is a kitchen actuator, is the brain command and control of the kitchen, catering enterprises or individuals According to their preferences in the "cloud" booking or ordering. This intelligent cooking robot can cook almost everything you like to eat, whether it's a simple potluck meal or a master's exquisite meal!

In the future kitchen, you can't see the busy handcutting, no chattering oil smoke, no noisy kitchen shadow, no horizontal condiment bottles and cans, no slippery sink floor, only two robotic arms operate silently and orderly, under the standard condiment kitchenware and tableware, according to menu instructions, grab and deliver, all kinds of auxiliary cooking. The robot hand silently completes instructions on the touch screen. Orderly, clean and quiet, steaming pot, occasionally crisp Ding Dang's prompt sound, a plate of master's fine dishes are continuously loaded, so that diners can eat in a relaxed and clean environment.
With the continuous upgrading of robotic technology, the future kitchen will not only be cooked by cooperative robots, but also the refrigerator will regularly remind the temporary food, report the expired food, and according to the amount forecast replenishment list, after confirmation or modification, direct orders will be placed and distributed by third parties. Intelligent kitchen, by cooperative robots to complete the replacement of chefs, the liberation of family cooks, this will be an inevitable and irreversible trend.

\section{Opportunity and Prospect of Chinese Food industrialization}

China's catering market is an industry with huge development space. One, the rapid growth of China's economy and the development strategy of further expanding domestic demand have injected new vitality into the sustained prosperity of the catering market; Two(11), the prosperity of catering industry has strongly stimulated consumption and stimulated domestic demand, and the virtuous circle situation has given new connotation and sufficient development opportunities to the traditional Chinese cooking industry.

\subsection{Saving Resources and Comprehensive Utilization}

The main ingredients of Chinese catering are fresh agricultural products, from the field to the table, the general loss will be about $50 \%$. If we do not count the waste of dining table, the loss in circulation is about $20 \%$, and the loss in restaurant processing is $20 \%$, but through industrialization, we can at least reduce the loss of $20 \%$ in restaurant processing. This is due to the high comprehensive utilization rate of food materials by factories, which is a trillion-level resource saving for a populous country like China. Japan's catering industry, the utilization rate of each cow can reach more than $90 \%$, different parts of the cattle are transported to different demand enterprises, leftover materials can be used to compress fat cattle, basically no waste part. This has played an important role in the utilization and protection of environment, resources and energy.

\subsection{Greatly Improve the Stability of Quality and Food Safety}

Under the industrialization of catering, from planting, harvesting to processing, fixed production bases can strictly control the production environment, thus changing the situation that the quality was determined by weather before. For example, in the most suitable season for picking, vegetables are picked at one time, and then through efficient and advanced liquid nitrogen freezing and other technical treatment, the nutrients of agricultural products are completely locked, so that everyone can eat at ease, food is the first necessity of the people, safety is the lifeline. 


\subsection{The Consumption Concept of Young People has Changed}

With the development of economy and the improvement of people's living standards, people have moved from food and clothing to enjoyment, and people's concept of dietary consumption has changed dramatically among young people. The new generation of life pressure, the tedious housework, especially cooking will not, also do not want to spend time, the traditional family cooking is gradually replaced by social catering services, in recent years, takeout single soaring, public canteens all over the streets and alleys, fewer and fewer people cook at home, the demand for food is getting higher and higher, where to eat, what to eat? It has become a big "meal annoyance" for Chinese people. The industrialization of catering will bring richer, more mellow and more convenient experience to meet people's demand for catering in the future.

\subsection{Labor Costs Force Enterprises to Transform Catering Robots or Will Erupt}

In recent years, with the reduction of labor force and the increase of labor costs, the cost of chefs in catering enterprises accounts for nearly $20 \%$ of the turnover. The flow of chefs in domestic catering enterprises is large, and they are generally small teams with chefs as the core. Therefore, a group of chefs will make the hotel in trouble immediately, affecting the business and reputation of enterprises. The business of Chinese food enterprises depends largely on the chef's skills and dishes, so for restaurant owners, the risk of employing people is very high, and robots do not have this risk at all. Nowadays, more and more American restaurants are considering choosing robots, which are popular because of their accuracy, timeliness, strong replication, fast production speed, low cost and low risk. The popularity of robots has also promoted the standardization of catering products.

\subsection{To Truly Achieve Precision Marketing}

The same dish or dish name, if there is no standard, in fact, it is difficult for consumers themselves to know what they prefer in the end, the so-called preferences, positioning is also vague, a diner who likes to eat Fish Head Pot, in all parts of the country to eat dishes called Fish Head Pot, from the selection of materials, side dishes, practices, tastes, plates, etc. All of these are different, even their own can not tell which one is good. After standardization and industrialization, diners can more accurately understand their preferences, but also allow enterprises to more accurate marketing and services, on this basis, but also for food and other agricultural products IP marketing to provide new ideas and channels, with a variety of condiments, accessories, additives, tableware and so on can be more accurate marketing.

\subsection{To Realize the International Spread of Chinese Food}

Chinese people have opened countless Chinese restaurants all over the world. Because Chinese food has not been baptized by industrialization, it has become the main obstacle to the chain and scale of Chinese food. Overseas more than 40 million Chinese or Chinese stomach, thousands of miles away from the Chinese difficult to eat, like Chinese food foreigners also have no happiness, Chinese food industrialization will be able to enter the Chinese market with Western food, in all parts of the world, enjoy incomparable Chinese cuisine, the Belt and Road strategy, Chinese cuisine is also a national soft power output. Let Chinese cooking really go to the international catering stage and let the whole world enjoy it.

\subsection{Non-Hereditary Inheritance of Chinese Cuisine}

The intangible cultural heritage of a nation retains the original state of its culture and its unique way of thinking. Intangible cultural heritage is not only the cultural symbol of a nation's existence, but also the cultural gene of a nation's development. Innovation of inheritance mode of intangible cultural heritage of catering category, focusing on the inheritance of technology and knowledge, recording the knowledge and skills of the holders and transforming them into "tangible" cultural heritage, attaching importance to the practical link of handicraft production, productive protection can stimulate the vitality of intangible cultural heritage of catering category, which is conducive to the transmission from generation to generation. The diet of a quarter of the world's people belongs to the category of Chinese cooking, and Chinese cooking technology is the first in the world. Chinese cooking and catering industry have gradually become one of the ways to carry forward the traditional culture of the Chinese nation, and also the carrier of enhancing national self-confidence and cultural self-confidence of Chinese people.

\subsection{After the Industrialization of Chinese Food, it can Build a Fully Automatic Intelligent Kitchen with the Whole Industry Chain}

In addition to solving the key robotic automatic cooking, the industrialized kitchen system also includes intelligent modules such as food storage and automatic cleaning, and connects all aspects of cooking through intelligent robotic arm, from meal preparation to cleaning, to realize the full automation of kitchen cooking scene. But a smart kitchen is not only to complete cooking and satisfy the taste, but also to compare your health testing data with digital recipes through big data, and combine nutritional data to tailor more humane and healthy recipes to different health states and different groups, and through cooking on temperature, oil and salt. Assist people with sub-health and chronic diseases to achieve health indicators. In addition, the future intelligent kitchen can also extend the 
industrial chain to both ends, standardize planting and clean vegetable pretreatment, and carry out cross-domain cooperation, which is also the core demand of the ecological influence construction of intelligent kitchen.

\subsection{Respect Masters and Non-Legacy Copyright, and Implement Knowledge Payment}

The menu database of the intelligent kitchen first saves the cooking methods, actions, processes, ingredients, seasonings and other big data of various chefs, various cuisines and various dishes. It can also constantly innovate and enrich the database. The intelligent cooking robot can constantly copy the dishes, which will play a great role in promoting the spread of Chinese food culture. After uploading and sharing, we can open the terminal to book or place orders anytime and anywhere, from home cooking to famous teachers' masterpieces, we can set different charging standards according to different levels, free or charging, just as we open an app, on-demand movies or songs, according to the number of on-demand, copyright owners will continue to have the platform's commission income, and realize pay as you order. The cooking industry also respects originality, encourages innovation and meets all kinds of needs as far as possible. In the future, the realization of "cloud cooking" in China, we can automatically complete cooking by choosing the recipes we want to eat from the cloud, realize crossregional dissemination, intergenerational inheritance, create cooking copyright, and realize knowledge payment.

\subsection{The spread of bacteria and viruses to promote the industrialization of catering}

With the emergence of COVID-19 epidemic, the physical catering industry has been greatly impacted, catering stores are facing reshuffling, and the takeout market will continue to grow in the next three years: It is estimated that the scale of Internet catering takeout in China will reach 598 billion yuan in 2020. This requires the industrialization of catering industry, avoiding more opportunities for human-to-human contact and infection, and providing strong protection and support for the health of regions and groups. In the future, new service scenarios such as community sharing, unmanned retail and so on can be realized through smart kitchen.

\section{Conclusions}

Cooperative robots break through the obstruction of Chinese cooking and make the industrialization of Chinese food possible, which is an important milestone in the innovation and upgrading of traditional catering industry. In the future, robot chefs and traditional chefs will coexist, but robot chefs will show great advantages in terms of large demand, high quality requirements, cost reduction, pursuit of scale effect, breaking through time and space constraints and restoring master classics. The industrialization of Chinese food will bring profound changes to the whole industry and industry chain, and science and technology will promote progress and change life.

\section{References}

1. Discussion on the Development Prospect and Trend of Chinese Cooperative RobotLiu Zhuang;- "Robot Industry"-2020-03-25

2. Discussion on the Development Prospect and Trend of Cooperative Robot in the Era of "Intelligent Economy" CCID Consultant-"China Computer News"-2020-06-29

3. "Synergy and Integration, Intelligence Leading to the Future"--2019 China Robot Industry Development Conference was successfully held in Chongqing"Instrument Standardization and Measurement"2019-12-26

4. Discussion on the Development of Chinese Food Industrialization Zhang Zhan-"Food Science and Technology"-2003-10-30

5. Cloud Robot: a New Model of Future Robot Wu Hongmin; Xu Zhihao; Zhou Songbin; Zhou Xuefeng;- "Guangdong Science and Technology"2020-08-15

6. Research and development of world-class cooperative robots based on independent brands"China Industry News"-2020-08-24 